\title{
The birth environment's effects on microbiome colonization and intestinal development
}

Selma-Royo M.

Calatayud Arroyo M.

García-Mantrana I

Parra-Llorca A.

Escuriet $\mathbf{R}$

Martínez-Costa C

Collado M C

\section{Video Byte}

Keywords: Microbiota, environment, mode of birth, antibiotics, epithelial barrier, immune system, early programming, innate immune system development, perinatal, home birth, caesarean section, vaginal birth, microbiome, colonization

Posted Date: February 25th, 2021

DOI: https://doi.org/10.21203/rs.3.rs-275916/v1

License: (a) (i) This work is licensed under a Creative Commons Attribution 4.0 International License. Read Full License 


\section{Abstract}

The microbial colonization of a newborn's intestine is a critical step in their development. and environmental factors during and after birth dramatically shape that colonization. Little is known about microbial colonization of infants born at home, outside the highly sanitized hospital environment. and the rate of home births is rapidly increasing in the United States but is still rare in Europe. New research examined the microbiomes of healthy infants born at home or in a hospital. Infants born in a hospital (CS and VAG) had greater microbial richness, or number of species, than infants born at home (HB). Infants born in the hospital via c-section (CS) had decreased Bacteroidetes and Bifidobacterium, along with a higher BMI and W/L during the first 18 months of life than the other groups. Different microbial profiles also had distinct effects on in vitro intestinal and immunological models. Specifically, adding the supernatant from home birth microbiota to the model systems resulted in a stronger epithelial barrier and increased immunological response compared to hospital-derived microbiota. This study shows a unique mechanistic link between microbial colonization factors and immune system development. The results could be used to adjust medical practices at birth to optimize bacterial colonization and ultimately improve immune system and intestinal development in neonates. 